\title{
BEST POSSIBLE APPROXIMATION CONSTANTS
}

\author{
BY
}

\author{
A. M. FINK
}

\begin{abstract}
We study inequalities between the norm of the best approximating polynomial and the $n$th derivative of the function. These inequalities are then related to inequalities that have been considered elsewhere in different contexts.
\end{abstract}

1. Introduction. Phillips [1] has shown that if $f^{(n+1)}$ is continuous and $P$ is a polynomial of degree $n$, then

$$
\left(\int_{-1}^{1}|f(x)-P(x)|^{p}\right)^{1 / p} \leqslant \frac{\delta(n, p)}{(n+1) !}\left\|f^{(n+1)}\right\|_{\infty}
$$

where $\delta(n, p)=\inf _{Q}\left(\int_{-1}^{1}\left|x^{n}-Q(x)\right|^{p}\right)^{1 / p}$ with $Q$ a polynomial of degree $(n-1)$. Furthermore, this is the best possible inequality of its kind.

We are interested in the generalizations of (1) where $\left\|f^{(n+1)}\right\|_{\infty}$ is to be replaced by a more general norm. Although such inequalities are of interest in themselves, our main interest is showing connections with inequalities that have been considered elsewhere, see Fink [2], [3] and Brink [4].

To set the precise problem, let $1 \leqslant p \leqslant \infty$ and $1 \leqslant q \leqslant \infty$. Let $\pi_{n}$ denote the polynomials of degree $\leqslant n$ and $f$ be a function with absolutely continuous $f^{(n)}$ and $f^{(n+1)} \in L_{q}(a, b)$. Let $P(f, n, p)$ be an element of $\pi_{n}$ that minimizes $\left\{\|f-Q\|_{p}: Q \in \pi_{n}\right\}$, and $E(f, n, p)=\|f-P(f, n, p)\|_{p}$. All norms are on the interval $[a, b]$. Then we seek best possible constants $B(n, p, q)$ such that

$$
E(f, n, p) \leqslant B(n, p, q)(b-a)^{n+1+1 / p-1 / q}\left\|f^{(n+1)}\right\|_{q} .
$$

The factor $(b-a)^{n+1+1 / p-1 / q}$ is inserted in order to make the constants $B(n, p, q)$ independent of the interval. The inequality (1) of Phillips then is the equation

$$
B(n, p, \infty)=\delta(n, p) /(n+1) ! 2^{n+1+1 / p} .
$$

It is known that

Received by the editors June 25, 1975 and, in revised form, November 7, 1975.

AMS (MOS) subject classifications (1970). Primary 41A10, 41A50; Secondary 41A05, 26A84. Key words and phrases. Best polynomial approximation.

- American Mathematical Society 1977 


$$
\delta(n, 1)=\delta(n, \infty)=1 / 2^{n} \text { and } \delta(n, 2)=\sqrt{2 /(2 n+3)} 2^{n+1} /\left(\begin{array}{c}
2+2 \\
n+1
\end{array}\right) .
$$

We will find it convenient to adopt $[-1,1]$ as the standard interval as in [1]. Also if $1 \leqslant p \leqslant \infty$, then $p^{\prime}$ is defined by $1 / p+1 / p^{\prime}=1$ with the usual conventions when $p=1$ or $\infty$. The norms $\|f\|=\left(\int_{-1}^{1}|f|^{p}\right)^{1 / p}$ and $\|f\|_{\infty}$ $=\operatorname{ess} \sup |f(x)|$ will be used.

2. The related inequalities. We here consider some special cases of inequalities considered in [2]. Let $f$ be defined on $[a, b]$ with $f^{(n-1)}$ absolutely continuous and $f^{(n)} \in L_{q}(a, b)$. The notation $f \in Z(\alpha, \beta)$ means that $f$ has at least an $\alpha$-fold zero at $a$ and a $\beta$-field zero at $b$. The notation $f \perp \pi_{n}$ means that $\int_{a}^{b} f q=0$ whenever $q \in \pi_{n}$. Let $D(n, p, q)$ and $F(n, p, q)$ be defined as the best possible constants in the inequalities,

$$
\|f\|_{p} \leqslant D(n, p, q)(b-a)^{n+1 / p-1 / q}\left\|f^{(n)}\right\|_{q}, \quad f \in Z(n, n) ;
$$

and

$$
\|f\|_{p}<F(n, p, q)(b-a)^{n+1 / p-1 / q}\left\|f^{(n)}\right\|_{q}, \quad|f|^{p-1} \operatorname{sgn}(f) \perp \pi_{n-1} .
$$

The inequality for (4) makes sense for all $p$ and $q$, while (5) requires that $p<\infty$.

For our purposes we need to know about the existence of solutions of the boundary value problems associated with inequalities (4) and (5).

LeMma 1. (i) If $g \in L_{1}(a, b)$ and $g \perp \pi_{n-1}$, then there is a unique $f \in Z(n, n)$ such that $f^{(n)}=g$ (a.e.). The unique $f$ is given by the formula

$$
f(x)=\frac{1}{(n-1) !} \int_{0}^{x}(x-t)^{n-1} g(t) d t .
$$

(ii) If $g \in L_{1}(a, b)$, then there is a unique function $f$ such that $f^{(n)}=g$ a.e. and $f \perp \pi_{n-1}$.

Proor. In the first instance a solution is given by the formula. The difference to two solutions is a function $p$ such that $p^{(n)}=0$ and $p \in Z(n, n)$. Clearly $p$ is a polynomial with too many zeros unless $p=0$.

In the second case, let $f_{0}$ be a particular solution of $f^{(n)}=g$. The general solution is thus given by $f_{0}+p$ with $p \in \pi_{n-1}$. Consider the problem $\min _{p \in \pi_{n-1}}\left\|f_{0}+p\right\|_{2}$. This finite dimensional problem has a solution $f_{0}-p_{0}$ $\equiv f_{1}$. The inequality $\left\|f_{1}\right\|_{2} \leqslant\left\|f_{1}+a p\right\|_{2}$ for arbitrary real $a$, and $p \in \pi_{n-1}$ leads to $\int_{-1}^{1} f_{1} p=0$ as required. Uniqueness now follows from $\left\|f_{1}\right\|_{2}$ $=\left\|f_{1}+p\right\|_{2}, p \in \pi_{n-1} \Rightarrow\left\|p^{2}\right\|_{2}=0$.

From the above representation it is easy to see that $D(n, p, q)$ exists. In fact, if $f \in Z(n, n)$ then an integration by parts shows that $f^{(n)} \perp \pi_{n-1}$ so that 


$$
D(n, p, q) \leqslant \frac{1}{(n-1) !}\left(\int_{a}^{b}\left(\int_{a}^{x}|x-t|^{(n-1) q^{\prime}} d t\right)^{p / q^{\prime}} d x\right)^{1 / p} .
$$

In general this is a poor estimate of $D(n, p, q)$.

LeMMA 2. If $p<\infty$ and $1<q$. Then $D\left(n, q^{\prime}, p^{\prime}\right)=F(n, p, q)$.

Proof. Let

$$
|f|^{p-1} \operatorname{sgn}(f) \perp \pi_{n-1} \quad \text { and } \quad h^{(n)}=|f|^{p-1} \operatorname{sgn}(f)
$$

with $h \in Z(n, n)$. Then

$$
\begin{aligned}
\|f\|_{p}^{p} & =\int_{a}^{b} h^{(n)} f=(-1)^{n} \int_{a}^{b} h f^{(n)} \leqslant\|h\|_{q^{\prime}}\left\|f^{(n)}\right\|_{q} \\
& \leqslant D\left(n, q^{\prime}, p^{\prime}\right)(b-a)^{n+1 / q^{\prime}-1 / p^{\prime}}\left\|h^{(n)}\right\|_{p^{\prime}}\left\|f^{(n)}\right\|_{q} \\
& =D\left(n, q^{\prime}, p^{\prime}\right)(b-a)^{n+1 / p-1 / q}\|f\|_{p}^{p-1}\left\|f^{(n)}\right\|_{q} .
\end{aligned}
$$

Thus $F(n, p, q)$ exists and is $\left\langle D\left(n, q^{\prime}, p^{\prime}\right)\right.$. Conversely, if $f \in Z(n, n)$, let $\boldsymbol{g}^{(n)}=|f|^{q^{\prime}-1} \operatorname{sgn}(f)$ with $g \perp \pi_{n-1}$. Then as before,

$$
\begin{aligned}
\|f\|_{q^{\prime}}^{q^{\prime}} & =(-1)^{n} \int_{a}^{b} f^{(n)} g \leqslant\|g\|_{p}\left\|f^{(n)}\right\|_{p^{\prime}} \\
& <F(n, p, q)(b-a)^{n+1 / p-1 / q}\left\|g^{(n)}\right\|_{q}\left\|f^{(n)}\right\|_{p^{\prime}} \\
& =F(n, p, q)(b-a)^{n+1 / p-1 / q}\|f\|_{q^{\prime}}^{q^{\prime}}\left\|f^{(n)}\right\|_{p^{\prime}}
\end{aligned}
$$

so that $D\left(n, q^{\prime}, p^{\prime}\right) \leqslant F(n, p, q)$.

Let us call functions for which equality holds in (4) or (5) extremals. The proof of Lemma 2 gives the equations that must be satisfied, since everything, say in the first string of inequalities, must be equalities. In particular, there are either extremals for both problems $\left(D\left(n, q^{\prime}, p^{\prime}\right), F(n, p, q)\right)$ or neither, and equality must hold in the use of Hölder's inequality. Thus extremal equations are

$$
\begin{aligned}
h^{(n)} & =|f|^{p-1} \operatorname{sgn}(f), & & |f|^{p-1} \operatorname{sgn}(f) \perp \pi_{n-1} ; \\
\left|f^{(n)}\right|^{q} & =\lambda^{2}|h|^{q^{\prime}}, & & h \in Z(n, n),
\end{aligned}
$$

for some constant $\lambda$. Here $h$ is an extremal for $D\left(n, q^{\prime}, p^{\prime}\right)$ and $f$ for $F(n, p, q)$.

Actually, it is easy to see that in most cases extremals do exist. Clearly $D(n, p, q)$ is $\sup \left\{\|f\|_{p}:\left\|f^{(n)}\right\|_{q}=1, f \in Z(n, n)\right\}$. If $\left\{f_{m}\right\}$ is a sequence of functions such that $\left\|f_{m}\right\|_{p} \rightarrow D(n, p, q)(b-a)^{n+1 / p-1 / q}$, and if $1<q<\infty$, then we can assume that $f_{m}^{(n)} \rightarrow g$ weakly with $\|g\|_{q}=1$. Thus 


$$
f_{m}(x)=\int_{a}^{x} \frac{(x-t)^{n-1}}{(n-1) !} f_{m}^{(n)}(t) d t
$$

converges uniformly to

$$
f(x)=\int_{a}^{x} \frac{(x-t)^{n-1}}{(n-1) !} g(t) d t .
$$

It follows that $f^{(n)}=g$ a.e., $f \in Z(n,-)$ and $\left\|f^{(n)}\right\|_{q}=1$ so that

$$
\|f\|_{p}=D(n, p, q)(b-a)^{n+1 / p-1 / q} \text {. }
$$

Since $f_{m}^{(n)} \perp \pi_{n-1}, g \perp \pi_{n-1}$. From this it follows that $f \in Z(n, n)$.

One can show that $D(n, p, q)$ is continuous in $p$ and $q$ and monotone in $p$ and $q$. In fact if $p<r,\|f\|_{p} \leqslant\|f\|_{r}(b-a)^{1 / p-1 / r}$ so that if $f \in Z(n, n)$, then

$$
\|f\|_{p} \leqslant\|f\|_{r}(b-a)^{1 / p-1 / r} \leqslant(b-a)^{1 / p-1 / r} D(n, r, q)(b-a)^{n+1 / r-1 / q}\left\|f^{(n)}\right\|_{q} .
$$

Since $D(n, p, q)$ is the best possible constant, it follows that $D(n, p, q)$ $<D(n, r, q)$ if $p<r$. Similarly, $D(n, p, q)$ is nonincreasing in $q$.

3. The main connection. The boundary conditions associated with inequality (5) are reminiscent of the properties of best possible approximation of functions by polynomials. We can make this precise. We will show that

THEOREM 1. For $1 \leqslant p<\infty, 1<q \leqslant \infty, B(n, p, q)=F(n+1, p, q)$.

In order to prove this, we need a preliminary observation.

LEMMA 3. $E(f, n, p)=\sup \left\{\int_{a}^{b}(f-P(f, n, p)) g: g \in L_{p^{\prime}}(a, b),\|g\|_{p^{\prime}}<1, g\right.$ $\left.\perp \pi_{n}\right\}$.

Proof. We have

$$
\|f-P(f, n, p)\|_{p}=\sup _{g} \int_{a}^{b}[f-P(f, n, p)] g \quad \text { where }\|g\|_{p^{\prime}}<1 .
$$

The supremum is attained for

$$
g=\lambda^{-1}|f-P(f, n, p)|^{p-1} \operatorname{sgn}(f-P(f, n, p))
$$

with $\lambda=\|f-P(f, n, p)\|_{p t}^{p}$. But this $g$ satisfies $g \perp \pi_{n}$, so that the supremum is attained among the more restrictive class of the lemma.

ProOF OF THE THEOREM. Let $g \in L_{p^{\prime}}(a, b), g \perp \pi_{n},\|g\|_{p^{\prime}} \leqslant 1$, and $G$ $\in Z(n+1, n+1)$ with $G^{(n+1)}=g$. Then 


$$
\begin{aligned}
\int_{a}^{b}[f-P(f, n, p)] g & =\int_{a}^{b} G^{(n+1)}[f-P(f, n, p)] \\
& =(-1)^{n+1} \int_{a}^{b} G f^{(n+1)} \leqslant\left\|f^{(n+1)}\right\|_{q}\|G\|_{q^{\prime}} \\
& \leqslant\left\|f^{(n+1)}\right\|_{q} D\left(n+1, q^{\prime}, p^{\prime}\right)(b-a)^{n+1+1 / q^{\prime}-1 / p^{\prime}}\left\|G^{(n+1)}\right\|_{p^{\prime}} \\
& =\left\|f^{(n+1)}\right\|_{q}(b-a)^{n+1+1 / p-1 / q} F(n+1, p, q)\left\|_{g}\right\|_{p^{\prime}} .
\end{aligned}
$$

And thus

$$
\|f-P(f, n, p)\|_{p} \leqslant(b-a)^{n+1+1 / p-1 / q} F(n+1, p, q)\left\|f^{(n+1)}\right\|_{q}
$$

so that $B(n, p, q) \leqslant F(n+1, p, q)$. Conversely, if $|f|^{p-1} \operatorname{sgn}(f) \perp \pi_{n}$, then $P(f, n, p)=0$ and

$$
\|f\|_{p}=E(f, n, p) \leqslant B(n, p, q)\left\|f^{(n+1)}\right\|_{q}(b-a)^{n+1 / p-1 / q}
$$

and, therefore, $F(n+1, p, q) \leqslant B(n, p, q)$.

There is an interplay between the various inequalities that we now want to explore. We now restrict ourselves to the standard interval $[-1,1]$. We first observe that Meinardus [5] notes that if $\left|f^{(n+1)}\right| \leqslant g^{(n+1)}$, then $E(f, n, p)$ $\leqslant E(g, n, p)$. Thus in order to prove an inequality (2) we can restrict ourselves to $f$ for which $f^{(n+1)} \geqslant 0$. In particular the extremals for problem (5) satisfy this relation and being $\perp \pi_{n}$ must have the minimum number of changes of sign. A glance at equations (6) then shows that $h^{(n)}$ has exactly $n$ changes of sign and $h$ is in $Z(n, n)$. Thus the extremal $h$ for inequality (4) satisfies $h \geqslant 0$.

To see that this is positive information we show that Phillips' result follows easily from this. For if $h \in Z(n, n)$ and $h \geqslant 0$, and $P \in \pi_{n}$ with $P^{(n)}=1$, then

$$
\|h\|_{1}=\int_{-1}^{1} h P^{(n)}=(-1)^{n} \int_{-1}^{1} h^{(n)} P \leqslant\left\|h^{(n)}\right\|_{q}\|P\|_{q^{\prime}}
$$

so that

$$
2^{n+1-1 / q} D(n, 1, q)=\min \left\{\|P\|_{q^{\prime}}: P \in \pi_{n}, P^{(n)} \equiv 1\right\}
$$

or

$$
2^{n+1-1 / q} D(n, 1, q)=\delta\left(n-1, q^{\prime}\right) / n !
$$

Therefore 
A. M. FINK

$$
B(n, p, \infty)=F(n+1, p, \infty)=D\left(n+1,1, p^{\prime}\right)=\frac{S(n, p)}{(n+1) ! 2^{n+1+1 / p}}
$$

4. $B(n, 1, q)$. In order to compute $B(n, 1, q)$ we note that we may assume that $f^{(n+1)}>0$. To see that this helps, we look at the extremal equations (6) and the proofs of Lemma 2 and Theorem 1. If $f$ is the extremal for the $F(n+1,1, q)$ pioblem, then $P(f, n, 1)=0$ and $f$ is also the extremal for the $B(n, 1, q)$ problem. Hence, there exists $h$, an extremal for the $D\left(n+1, q^{\prime}, \infty\right)$ problem, such that

$$
\begin{aligned}
& \operatorname{sgn}(f) \perp \pi_{n} ; \quad h^{(n+1)}=\operatorname{sgn}(f) ; \\
& h \in Z^{2}(n+1, n+1) ; \quad f^{(n+1)}=\lambda^{2 / q}|h|^{q^{\prime} / q} .
\end{aligned}
$$

Knowing that $f^{(n+1)} \geqslant 0$ it follows that $\operatorname{sgn}(f)$ changes sign exactly $(n+1)$ times. Thus $h^{(n+1)}$ changes sign exactly $(n+1)$ times. With $h$ already having $(2 n+2)$ zeros, we conclude that $h \neq 0$ on $(-1,1)$. Thus $f^{(n+1)}>0$ on $(-1,1)$. This implies, see Lorentz [6, p. 115], that the zeros of $f \operatorname{are} \cos (k \pi /(n+2)) ; k$ $=1, \ldots, n+1$. That is, $h$ is a perfect spline with knots at the Chebyshev points. According to Schoenberg [7], $h(x)=M(x) / 2^{n}(n+1)$ ! where $M$ is the basic spline $M\left(x ; x_{0}, \ldots, x_{n+2}\right) ; x_{i}=\cos (i \pi /(n+2))$. Therefore

TheOREM 2. $B(n, 1, q)=\left(1 / 2^{2 n+2-1 / q}\right)\|M\|_{q^{\prime}} /(n+1)$ !.

5. $B(n, 2, q)$. The computation of $B(n, 2, q)$ is more complicated. However, we may reduce it to several related problems. Since we know that $B(n, 2, q)$ $=F(n+1,2, q)$ we concentrate on computing $F(n, 2, q)$. The first step in this direction is

LEMMA 4. Let $g$ be continuous. Then $g=f^{(k)}$ for some $f \in Z(k, k)$ if and only if $g \perp \pi_{k-1}$.

Proof. If $g \perp \pi_{k-1}$, define

$$
f(x)=\int_{-1}^{x} \frac{(x-t)^{k-1}}{(k-1) !} g(t) d t .
$$

Then $f^{(k)}=g$ and $f$ has $k$ zeros at -1 . On the other hand,

$$
f^{(j)}(1)=\int_{-1}^{1} \frac{(1-t)^{k-1-j}}{(k-1-j) !} g(t) d t=0
$$

for $0 \leqslant k-1-j \leqslant k-1$. This gives $g \in Z(k, k)$. Conversely, if $g=f^{(k)}$, $f \in Z(k, k)$ then 


$$
\int_{-1}^{1} p(x) g(x) d x=\int_{-1}^{1} p(x) f^{(k)}(x) d x=(-1)^{k} \int_{-1}^{1} p^{(k)} f=0 \text { if } p \in \pi_{k-1} \text {. }
$$

Thus the inequality

$$
\|g\|_{2}<F(n, 2, q) 2^{n+1 / 2-1 / q}\left\|g^{(n)}\right\|_{q} ; \quad g \perp \pi_{n-1},
$$

is equivalent to

$$
\left\|f^{(n)}\right\|_{2}<F(n, 2, q) 2^{n+1 / 2-1 / q}\left\|f^{(2 n)}\right\|_{q} ; \quad f \in Z(n, n) .
$$

In order to see how this representation can be used, we introduce another class of inequalities. Consider

$$
\|f\|_{p} \leqslant C(n, \alpha, p, q)(b-a)^{n+1 / p-1 / q}\left\|f^{(n)}\right\|_{q}
$$

when $f \in Z(\alpha, n-\alpha)$.

These have been discussed in Brink [4] and Fink [2]. For our purposes here, we note that (see Fink [2]) for the problem $C\left(2 n, n, q^{\prime}, q\right)$, the extremal equations are

$$
\begin{aligned}
\left|f^{(2 n)}\right|^{q} & =\lambda^{2} \mid h^{q^{\prime}}, & & f \in Z(n, n), \\
h^{(2 n)} & =|f|^{q^{\prime}-1} \operatorname{sgn}(f), & & h \in Z(n, n),
\end{aligned}
$$

with $f^{(2 n)} \geqslant 0,(-1)^{n} h^{(2 n)} \geqslant 0, f$ the extremal for $C\left(2 n, n, q^{\prime}, q\right)$ and $h$ an extremal for the same problem.

The second fact that we need is the uniqueness of the extremals.

We postpone the proof in order to avoid interrupting the flow of events.

LEMMA 5. If $2<q<\infty$ then there is a unique extremal (up to multiplicative constant) for the $C\left(2 n, n, q^{\prime}, q\right)$ problem.

Since both $h$ and $f$ in (9) are in $Z(n, n)$ and the two functions appear in a symmetric way (aside from a multiplicative constant), we can conclude that $h(t)=\mu f(t)$ and

$$
f^{(2 n)}(t)=\lambda^{2 / q} h(t)^{q^{\prime} / q}=\left(\lambda^{2} \mu^{q^{\prime}}\right)^{1 / q} f(t)^{q^{\prime / q}} .
$$

THEOREM 3. $F(n, 2, q)=C\left(2 n, n, q^{\prime}, q\right)^{1 / 2}$ if $2<q<\infty$. If $f$ is the extremal for the $C\left(2 n, n, q^{\prime}, q\right)$ problem, then $f^{(n)}$ is the extremal for the $F(n, 2, q)$ and $B(n-1,2, q)$ problems.

Proor. Using Lemma 4, we let $f \in Z(n, n)$ and compute $\int_{-1}^{1}\left|f^{(n)}\right|^{2}$. 


$$
\begin{aligned}
\int_{-1}^{1}\left(f^{(n)}\right)^{2} & =(-1)^{n} \int_{-1}^{1} f f^{(2 n)} \leqslant\|f\|_{q^{\prime}}\left\|f^{(2 n)}\right\|_{q} \\
& <C\left(2 n, n, q^{\prime}, q\right) 2^{2 n+1 / q^{\prime}-1 / q}\left\|f^{(2 n)}\right\|_{q}^{2} .
\end{aligned}
$$

Thus

$$
\left\|f^{(n)}\right\|_{2} \leqslant C\left(2 n, n, q^{\prime}, q\right)^{1 / 2} 2^{n+1 / 2-1 / q}\left\|f^{(2 n)}\right\|_{q} .
$$

Consequently, $F(n, 2, q)<C\left(2 n, n, q^{\prime}, q\right)^{1 / 2}$. However, in inequalities (11), equality holds if $|f|^{q^{\prime}}=\sigma\left|f^{(2 n)}\right|^{q}$ a.e., $\sigma$ some constant, and if $f$ is an extremal for $C\left(2 n, n, q^{\prime}, q\right)$. By $(10)$ both of these are satisfied. Therefore, $F(n, 2, q)$ $=C\left(2 n, n, q^{\prime}, q\right)^{1 / 2}$.

We can combine this with the extremal information if $q=2$ to get more information.

THEOREM 4. $B(n, 2,2)=\lambda_{0}^{-1 / 2}$ where $\lambda_{0}$ is the smallest eigenvalue of the problem

$$
f^{(2 n+2)}=\lambda(-1)^{n+1} f, \quad f^{(j)}(0)=f^{(j)}(1)=0, \quad j=0, \ldots, n .
$$

Proor. By Theorem 3 we may compute $C(2 n+2, n+1,2,2)^{1 / 2}$ instead. By Lemma 5 the extremal is unique. The extremal equations are

$$
\begin{array}{lll}
h^{(2 n+2)}=f, & & h \in Z(n+1, n+1), \\
f^{(2 n+2)}=\sigma(-1)^{n+1} h, & & f \in Z(n+1, n+1),
\end{array}
$$

with $f$ the extremal. But $h$ is obviously also an extremal so that $h=\mu f$ for some $\mu>0$. That is (12) is satisfied. But

$$
C(2 n+2, n+1,2,2)=\|f\|_{2} /\left\|f^{(2 n)}\right\|_{2}=\|f\|_{2} / \lambda\|f\|_{2}=1 / \lambda
$$

so that the result follows.

6. Proof of the uniqueness lemma.

Proof of Lemama 5. The extremal equations (9) may be simplified in the following way. Since $f^{(2 n)}>0$ and $f \in Z(n, n), f$ has no interior zeros. A similar statement holds for $h$. Hence

$$
f^{(2 n)}(x)=\lambda^{2 / q}|h|^{q^{\prime}-1} \text { and } h^{(2 n)}(x)=|f|^{q^{\prime}-1} .
$$

Let $G(t, s)$ be the Green's function for the problem $L y=y^{(2 n)}, y \in Z(n, n)$. It is known that $(-1)^{n} G(t, s) \geqslant 0$. Now the extremal equations may be written

$$
f(x)=\lambda^{2 / q} \int_{-1}^{1}(-1)^{n} G(x, s)|h(s)|^{q^{\prime}-1} d s,
$$




$$
h(x)=\int_{-1}^{1}(-1)^{n} G(x, s)|f(s)|^{q^{\prime}-1} d s .
$$

Thus $f$ is an eigenfunction of the operator $K$ which is a composition of operators $H_{1}$ and $H_{2}$ where

$$
H_{1} f(x)=(-1)^{n} \int_{-1}^{1} G(x, t) f(t) d t \text { and } H_{2} f(x)=|f(x)|^{q^{\prime}-1} .
$$

In fact, if $K f(x)=\left(H_{1} H_{2} H_{1} H_{2}\right) f$, then $f>0$ is a nonnegative solution to $f=\lambda^{2 / q} K f$. Furthermore since $H_{1}$ and $H_{2}$ leave the cone $\{f \mid f>0\}$ invariant, $K$ is a positive operator with respect to this cone.

Using equations (9), the normalization $\left\|f^{(2 n)}\right\|_{q}=1$, and

$$
C\left(2 n, n, q^{\prime}, q\right) 2^{n+1 / q^{\prime}-1 / q}=\|f\|_{q^{\prime}}=\|h\|_{q^{\prime}} /\left\|h^{(2 n)}\right\|_{q},
$$

it follows that

$$
C\left(2 n, n, q^{\prime}, q\right) 2^{n+1 / q^{\prime}-1 / q}=\lambda^{-2 / q q^{\prime}} .
$$

Consequently, the uniqueness of extremals amounts to the statement that the integral equation $f=\lambda^{2 / q} K f$ with $\lambda$ given by (13) has a unique solution when $\left\|f^{(2 n)}\right\|_{q}=1$. It is easy to verify that the operator $K$ is a $u_{0}$-concave monotone operator in the sense of Krasnoselskii [8, pp. 187-188] and so there is at most one nonnegative solution $f$ to $f=\lambda^{2 / q} K f$ provided $u_{0}=x^{n}$ and $0<q^{\prime}-1$ $<1$. If $q^{\prime}=2$, the result follows from linear theory.

7. On $B(n, \infty, q)$ and $B(n, p, 1)$. As a consequence of Lemma 2 and Theorem 1, $B(n, p, q)=D\left(n+1, q^{\prime}, p^{\prime}\right)$ whenever $1<p<\infty$ and $1<q<\infty$. In fact, the equation is true for all $p$ and $q$ in $[1, \infty]$. We have shown that $D$ is increasing in its second variable and decreasing in its third variable. Consequently $B$ has the same properties.

LEMasA 6. $D$ and $B$ are continuous from the left in the second variable and continuous from the right in the third variable.

Proof. Let $1<p \leqslant \infty$ and $1<p_{1}<p$, and $[a, b]=[0,1]$. If $f$ is any admissible function, then

$$
\|f\|_{p_{1}} \leqslant D\left(n, p_{1}, q\right)\left\|f^{(n)}\right\|_{q} \leqslant D(n, p-, q)\left\|f^{(n)}\right\|_{q}
$$

by the monotonicity of $D$. Now letting $p_{1} \rightarrow p$ we get $\|f\|_{p}<D(n, p-, q)$ - $\left\|f^{(n)}\right\|_{q}$. By minimality, $D(n, p, q)<D(n, p-, q)$. By monotonicity the reverse is true, so $D(n, p, q)=D(n, p-, q)$. The proof for $B$ and for the third variable are similar.

TheOREM 5. For $1 \leqslant p \leqslant \infty$ and $1 \leqslant q \leqslant \infty, B(n, p, q)=D\left(n+1, q^{\prime}, p^{\prime}\right)$. 
Proof. This is true if $p \neq \infty$ and $q \neq 1$. However, letting $p \lambda \infty$, we have $p^{\prime} \searrow 1$ and by the lemma, $B(n, \infty, q) \equiv D\left(n+1, q^{\prime}, 1\right)$. The other cases are similar.

In particular, we are interested here in the equality

$$
B(n, \infty, q)=D\left(n+1, q^{\prime}, 1\right)
$$

and estimates for these numbers. The interest stems from the string of inequalities:

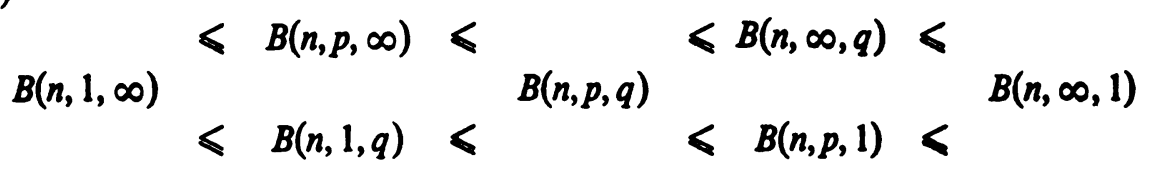

which hold for $1<p \leqslant \infty$ and $1 \leqslant q \leqslant \infty$. We already know that

$$
B(n, 1, \infty)=\delta(n, 1) /(n+1) ! 2^{n+2}=1 /(n+1) ! 2^{2 n+2}
$$

giving a lower bound for $B(n, p, q)$. Furthermore the numbers $B(n, p, \infty)$ and $B(n, 1, q)$ are nicely characterized and are more general lower bounds. We would like to similarly characterize the numbers $B(n, \infty, q)$ and $B(n, p, 1)$.

We deal with the numbers $B(n, p, 1)$ first.

\section{THEOREM 6.}

$$
B(n, p, 1)=\frac{1}{2^{n+1+1 / p}} \max _{x_{0}} \min _{r \in \pi_{n}}\left\|_{g}+r\right\|_{p}
$$

where $g^{(n)}=\operatorname{sgn}\left(x-x_{0}\right)$ and the norm is on the interval $[-1,1]$.

Proor. We begin by computing $D\left(n, \infty, p^{\prime}\right)$. Let $f \in Z(n, n)$.

There is an $x_{0} \in(-1,1)$ such that $f^{\prime}\left(x_{0}\right)=0$ and $\|f\|_{\infty}=\left|f\left(x_{0}\right)\right|$. Then for $g^{(n-1)}=\operatorname{sgn}\left(x-x_{0}\right)$

$$
\|f\|_{\infty}=\left|f\left(x_{0}\right)\right| \leqslant \frac{1}{2} \int_{-1}^{1}\left|f^{\prime}\right|= \pm \frac{1}{2} \int_{-1}^{1} f^{\prime} g^{(n-1)}= \pm \frac{1}{2} \int_{-1}^{1} f^{(n)} g
$$

by an integration by parts. Now since $f \in Z(n, n), f^{(n)} \perp \pi_{n-1}$ so we have for any $r \in \pi_{n-1}$,

$$
\|f\|_{\infty} \leqslant \pm \frac{1}{2} \int_{-1}^{1} f^{(n)}(g+r)
$$

and thus

$$
\|f\|_{\infty}<\frac{1}{2}\|g+r\|_{p}\left\|f^{(n)}\right\|_{p^{\prime}}
$$


with equality when $\left|f^{(n)}\right|^{p^{\prime}}=\lambda^{2}|g+r|^{p}$ a.e. for some $\lambda$. However, in order to be admissible, $f^{(n)} \perp \pi_{n-1}$. If we now select $r$ so that $g+r$ minimizes $\left\|_{g}+r\right\|_{p}$ then $|g+r|^{p-1} \operatorname{sgn}(g+r) \perp \pi_{n-1}$ so that $f^{(n)} \perp \pi_{n-1}$ and $f$ may be an extremal. In order to make the inequality hold for all $f$ we need to maximize over the choice of $x_{0}$. Thus

$$
D\left(n, \infty, p^{\prime}\right) 2^{n-1 / p^{\prime}}=\max _{x_{0}} \min _{r \in \pi_{n-1}}\|g+r\|_{p}
$$

with $g^{(n-1)}=\operatorname{sgn}\left(x-x_{0}\right)$. Now $B(n, p, 1)=D\left(n+1, \infty, p^{\prime}\right)$ and the proof is complete.

If it were known that the extremal $f$ is unique then clearly $f$ would be even and $x_{0}=0$ and one would not have to maximize over $x_{0}$. It is possible that for $p=\infty$ that $g+r$ is the perfect spline which is called the Chebyshev-Euler spline $t_{n, 1}$ in Schoenberg [9].

Although the above theorem gives an algorithm for finding $B(n, p, 1)$ we cannot obtain any explicit numbers. However, we can show that

$$
B(n, p, 1)<\delta(n-1, p) / n ! 2^{n+1+1 / p} .
$$

To see this, let $f \in Z(n, n)$. Then, as above,

$$
\|f\|_{\infty}<\frac{1}{2}\left\|f^{\prime}\right\|_{1}<\frac{1}{2} D\left(n-1,1, p^{\prime}\right) 2^{n-1 / p^{\prime}}\left\|f^{(n)}\right\|_{p^{\prime}}
$$

so that

$$
\begin{aligned}
D\left(n, \infty, p^{\prime}\right) 2^{n-1 / p^{\prime}} & <D\left(n-1,1, p^{\prime}\right) 2^{n-1-1 / p^{\prime}} \text { or } \\
D\left(n, \infty, p^{\prime}\right) & <\frac{1}{2} D\left(n-1,1, p^{\prime}\right) .
\end{aligned}
$$

Thus

$$
\begin{aligned}
B(n-1, p, 1) & =D\left(n, \infty, p^{\prime}\right)<\frac{1}{2} D\left(n-1,1, p^{\prime}\right)=\frac{1}{2} B(n-2, p, \infty) \\
& =\frac{\delta(n-2, p)}{2(n-1) ! 2^{n-1+1 / p}}
\end{aligned}
$$

so that (15) follows. In particular,

$$
\frac{1}{(n+1) ! 2^{2 n+2}}=B(n, 1, \infty)<B(n, p, q)<B(n, \infty, 1)<\frac{1}{n ! 2^{2 n}} .
$$

It is probably not true that equality holds in (15) since in the proof it is not likely that both inequalities can be equalities. The extremal for $D\left(n-1,1, p^{\prime}\right)$ should have no interior zero as $f^{\prime}$ does. 


\section{THEOREM 7.}

$$
B(n, \infty, q)=\min _{x_{1}} \max _{x} \frac{\left|\omega_{n}(x)\right|\|M(\cdot, x)\|_{q^{\prime}}}{2^{n-1 / q}}
$$

where $\omega_{n}(x)=\prod_{i=0}^{n}\left(x-x_{i}\right),-1 \leqslant x_{i} \leqslant 1$, and

$$
M(t, x)=M\left(t, x, x_{0}, \ldots, x_{n}\right)
$$

is the basic spline with knots at $x, x_{0}, \ldots, x_{n}$ (see Schoenberg [9]).

PROOF. It is known that if $P \in \pi_{n}$ interpolates $f$ at $x_{0}, \ldots, x_{n}$ then

$$
(f-P)(x)=\omega_{n}(x) \int_{-1}^{1} M(t, x) f^{(n)}(t) d t .
$$

Thus

$$
\|f-P\|_{\infty}<\max _{x}\left(\left|\omega_{n}(x)\right|\|M(\cdot, x)\|_{q^{\prime}}\right)\left\|f^{(n)}\right\|_{q} .
$$

If $\max$ of $\left|\omega_{n}(x)\right|\|M(\cdot, x)\|_{q^{\prime}}$ is attained at $x_{0}$ then (17) is an equality if $\left|f^{(n)}(t)\right|^{q}=\lambda^{2}\left|M\left(t, x_{0}\right)\right|^{q^{\prime}}$. In particular, $E(f, n, \infty)$ is dominated by the righthand side of (17) for every choice of $x_{i}$, hence, in particular, for the min over $x_{i}$. Thus $B(n, \infty, q)$ is as given.

For $q=\infty,\|M(\cdot, x)\|_{q^{\prime}}=\|M(\cdot, x)\|_{i}=1$ so that this agrees with the previous result. It should be possible to compute $B(n, \infty, 1)$ from this result but we have not been able to do it.

8. Other estimates. Besides the already quoted results, we also note some other scattered results. Using Theorem 3, we see that

$$
B(n, 2, q)=C\left(2 n+2, n+1, q^{\prime}, q\right)^{1 / 2} .
$$

If $q=\infty$, then

$$
B(n, 2, \infty)=((n+1) ! /(2 n+2) !)(2 n+3)^{-1 / 2}
$$

agrees with $C(2 n+2, n+1,1, \infty)$ given in [4]. Also

$$
B(n, 2,1)=C(2 n+2, n+1, \infty, 1)^{1 / 2}=\left(n ! 2^{2 n+1}\right)^{-1}(2 n+1)^{-1 / 2} .
$$

This last computation is an actual computation of the maximum of the Green's function (Brink [10]). Thus

$$
\frac{(n+1) !}{(2 n+2) !} \frac{1}{\sqrt{2 n+3}} \leqslant B(n, 2,2) \leqslant \frac{1}{n ! 2^{2 n+1}} \frac{1}{\sqrt{2 n+1}}
$$




\section{REFERENCES}

1. G. M. Phillips, Error estimates for best polynomial approximations, Approximation Theory (Proc. Sympos., Lancaster, 1969), Academic Press, London, 1970, pp. 1-6. MR 43 \# 3703.

2. A. M. Fink, Conjugate inequalities for functions and their derivatives, SIAM J. Math. Anal. 5 (1974), 399-411. MR 49 \#7407.

3. - Differential inequalities and disconjugacy, J. Math. Anal. Appl. 49 (1975), 758-772.

4. J. Brink, Inequalities involving $\|f\|_{p}$ and $\left\|f^{(n)}\right\|_{q}$ for $f$ with $n$ zeros, Pacific J. Math. 42 (1972), 289-311.

5. G. Meinardus, Approximation of functions, Springer, Berlin, 1964; English transl., SpringerVerlag, New York, 1967. MR 31 \#547; 36 \#571.

6. G. G. Lorentz, Approximation of functions, Holt, Rinehart and Winston, New York, 1966. MR 35 \# 4642; erratum, 36, p. 1567.

7. I. J. Schoenberg, The perfect B-splines and a time-optional control problem, Israel J. Math. 10 (1971), 261-274. MR 47 \#9130.

8. M. A. Krasnosel'skii, Positive solutions of operator equations, Fizmatgiz, Moscow, 1962; English transl. Noordhoff, Groningen, 1964. MR 26 \#2862; 31 \#6107.

9. I. J. Schoenberg, Cardinal spline interpolation, SIAM Regional Conf. Ser. Appl. Math., no. $12,1973$.

10. J. Brink, Private communication.

Department of Mathematics, Iowa State University, Ames, Iowa 50010 